MATHEMATICS OF COMPUTATION

Volume 74, Number 252, Pages 1937-1952

S $0025-5718(05) 01734-5$

Article electronically published on March 15, 2005

\title{
POLYNOMIAL SERIES EXPANSIONS FOR CONFLUENT AND GAUSSIAN HYPERGEOMETRIC FUNCTIONS
}

\author{
W. LUH, J. MÜLLER, S. PONNUSAMY, AND P. VASUNDHRA
}

\begin{abstract}
Based on the Hadamard product of power series, polynomial series expansions for confluent hypergeometric functions $M(a, c ; \cdot)$ and for Gaussian hypergeometric functions $F(a, b ; c ; \cdot)$ are introduced and studied. It turns out that the partial sums provide an interesting alternative for the numerical evaluation of the functions $M(a, c ; \cdot)$ and $F(a, b ; c ; \cdot)$, in particular, if the parameters are also viewed as variables.
\end{abstract}

\section{INTRODUCTION}

Many special functions are defined by Taylor expansion (in most cases around the origin). So a very direct way for numerically evaluating such functions is to take the partial sums of the Taylor series as an approximation. However, it turns out that in many cases - even for entire functions - Taylor sections provide a satisfactory accuracy only in a more or less small neighborhood of the origin. This is mainly due to cancellation errors occurring for arguments of larger modulus, in particular, if the function values are comparably small in modulus. So in most cases a satisfactory accuracy for a wider range of arguments is only achieved by using alternative methods.

One possibility is to replace partial sums of the Taylor series by partial sums of (suitably shifted) Chebyshev expansions. While often numerically much more stable, the Chebyshev expansions have the disadvantage that in many cases the coefficients themselves involve certain special functions, and so the computation of these coefficients turns out to be nontrivial.

In this paper we apply convolution techniques to study polynomial series expansions for certain classes of special functions which, on the one hand, behave numerically more stable than Taylor series and which, on the other hand, are comparably easy to compute. The general approach leads to series expansions, in particular, for the confluent hypergeometric functions, defined by

$$
M(a, c ; z)=\sum_{\nu=0}^{\infty} \frac{(a)_{\nu}}{(c)_{\nu}} \frac{z^{\nu}}{\nu !} \quad(z \in \mathbb{C})
$$

Received by the editor December 3, 2003 and, in revised form, May 18, 2004.

2000 Mathematics Subject Classification. Primary 33C05, 33C15, 33F05, 65D20.

Key words and phrases. Hypergeometric series, Hadamard product, polynomial expansions.

The work of the authors was supported by DST-DAAD under Project Based Personal Exchange Programme (Sanction No. INT/DAAD/P-64/2002). 
for $a, c \in \mathbb{C}, c \neq 0,-1,-2, \ldots$, where $(a)_{\nu}:=a(a+1) \cdots(a+\nu-1),(a)_{0}:=1$ is Pochhammer's symbol, and for the Gaussian hypergeometric functions,

$$
F(a, b ; c ; z)={ }_{2} F_{1}(a, b ; c ; z):=\sum_{\nu=0}^{\infty} \frac{(a)_{\nu}(b)_{\nu}}{(c)_{\nu}} \frac{z^{\nu}}{\nu !} \quad(|z|<1) .
$$

The method used in finding series representations is based on the Hadamard product $\phi * \psi$ of two power series, $\phi(z)=\sum_{n=0}^{\infty} a_{n} z^{n}$ and $\psi(z)=\sum_{n=0}^{\infty} b_{n} z^{n}$, defined as

$$
(\phi * \psi)(z)=\sum_{n=0}^{\infty} a_{n} b_{n} z^{n}
$$

Since this product turns out to have a convolution integral representation under suitable curves $\gamma$ in the complex plane $\mathbb{C}$, it is also called convolution product. More precisely, we have

Lemma 1.1. Let $S \subset \mathbb{C}$ be a region, which is starlike with respect to the origin, and let $\phi$ be holomorphic in the cut plane $\mathbb{C} \backslash[1, \infty)$. Suppose further that $K \subset S$ is compact and that $\gamma$ is a piecewise smooth Jordan curve in $\mathbb{C} \backslash[1, \infty)$ surrounding 0 and so that $K \cdot \gamma^{-1}:=\{z / \zeta: z \in K, \zeta \in \gamma\} \subset S$. If $\psi$ is holomorphic in $S$, then $\phi * \psi$ is analytically continuable into $S$ and we have, for every $z \in K$,

$$
(\phi * \psi)(z)=\frac{1}{2 \pi i} \int_{\gamma} \phi(\zeta) \psi\left(\frac{z}{\zeta}\right) \frac{d \zeta}{\zeta} .
$$

The proof is essentially an application of the Cauchy integral formula (cf., e.g., [4] or [7, for an even more general version).

If $K \subset \mathbb{C}$ is a compact subset and if we define

$$
\|\phi\|_{K}=\sup _{z \in K}|\phi(z)|
$$

for all functions $\phi$ continuous in $K$, then Lemma 1.1 implies the following continuity property of the Hadamard multiplication (the proof is similar to the one of Theorem 1 in $[8]$ ).

Theorem 1.3. Let $S$ be a region in $\mathbb{C}$, which is starlike with respect to the origin, and suppose that $\phi$ is holomorphic in $\mathbb{C} \backslash[1, \infty)$. If $K$ is a compact set in $S$, which is starlike with respect to the origin, then for every compact set $L \subset S$ with $K \subset L$ there exists a constant $M_{L}=M_{L}(\phi, K)>0$ such that for every function $\psi$ holomorphic in $S$ we have

$$
\|\phi * \psi\|_{K} \leq M_{L} \cdot\|\psi\|_{L} .
$$

The basic idea now is the following. Suppose we have been given a family $\mathcal{F}$ of functions (as, for example, the functions $M(a, c ; \cdot)$ or the functions $F(a, b ; c ; \cdot))$ and a fixed function $g$ that is holomorphic in a region $S$, being starlike with respect to the origin. Moreover, suppose that for every $f \in \mathcal{F}$ a function $\phi_{f}$ exists that is holomorphic in the cut plane $\mathbb{C} \backslash[1, \infty)$ and so that

$$
f=\phi_{f} * g .
$$

Now, if $\sum a_{n} P_{n}$ is a series expansion of $g$ (with polynomials $P_{n}$ of degree $\leq n$, say) that converges locally uniformly in $S$, then, according to the above continuity property of the Hadamard product, we have

$$
f=\sum a_{n}\left(\phi_{f} * P_{n}\right) \quad(f \in \mathcal{F})
$$


locally uniformly in $S$. In this way we obtain a series expansion for every $f \in \mathcal{F}$ (in polynomials $\phi_{f} * P_{n}$ of degree $\leq n$ ) with coefficients $a_{n}$ being independent of $f$.

\section{MAIN THEOREM}

In order to obtain more precise results on the behavior of the series as above we consider a restricted situation: From now on we suppose that the functions $\phi_{f}$ are hypergeometric functions $F(a, b ; c ; \cdot)$. Then the $\phi_{f}$ are, in particular, holomorphic in $\mathbb{C} \backslash[1, \infty)$. Our aim is to find, for polynomials $P$ of degree $\leq n$, an estimate for $\left\|\phi_{f} * P\right\|_{[0,1]}=\|F(a, b ; c ; \cdot) * P\|_{[0,1]}$ in terms of $\|P\|_{[0,1]}$.

For the proof of the main result we need to estimate the integrals of $|F(a, b ; c ; \zeta)| /|\zeta|$ on curves of the kind occurring in Lemma 1.1.

We consider the function

$$
\Phi(z)=4 z /(1+z)^{2},
$$

which maps the outer of the closed unit disk conformally onto $\mathbb{C} \backslash[1, \infty)$ with $\Phi(\infty)=0$. For $R>1$ let

$$
\gamma_{R}=\left\{\Phi\left(R e^{-i \theta}\right): \theta \in[-\pi, \pi]\right\}
$$

denote the image of the circle $|z|=R$ under the mapping $\Phi$ with positive orientation. (Then the curves $\gamma_{R}$ are as in Lemma 1.1 and, moreover, the curve $\gamma$ in Lemma 1.1 can always be chosen to be of the form $\gamma_{R}$, for $R$ depending on $K$.) If $R$ is close to 1 , there is exactly one point of intersection of $\gamma_{R}$ and $|z-1|=1 / 2$ in the upper half-plane (see Figure 2.1). Let $\theta_{1}=\theta_{1}(R)$ be the argument of the inverse image under $\Phi$ of this point (a computation shows that $\theta_{1}(R)=\arccos (1 /(6 R)+R / 6)$ for $R<3+2 \sqrt{2}$ ).

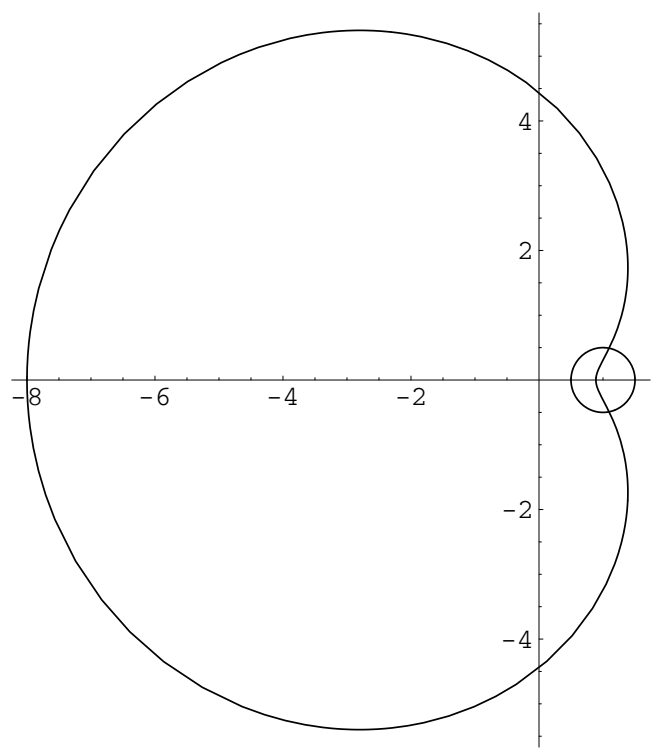

Figure 2.1. Curve $\gamma_{2.0}$ and circle $|z-1|=1 / 2$ 
Lemma 2.1. With the above notation we have

$$
\begin{aligned}
\int_{\gamma_{R}}|F(a, b ; c ; \zeta)| \frac{|d \zeta|}{|\zeta|} & \leq A \cdot \int_{0}^{\theta_{1}}\left[\frac{R^{2}+1-2 R \cos \theta}{R^{2}+1+2 R \cos \theta}\right]^{1 / 2} d \theta \\
& +B \cdot \int_{0}^{\theta_{1}}\left[\frac{R^{2}+1-2 R \cos \theta}{R^{2}+1+2 R \cos \theta}\right]^{\operatorname{Re}(c-a-b+1 / 2)} d \theta \\
& +C \cdot \int_{\theta_{1}}^{\pi} \frac{(4 R)^{-\operatorname{Re}(a)} \sqrt{R^{2}+1-2 R \cos \theta}}{\left(R^{2}+1+2 R \cos \theta\right)^{\operatorname{Re}(-a)+1 / 2}} d \theta \\
& +D \cdot \int_{\theta_{1}}^{\pi} \frac{(4 R)^{-\operatorname{Re}(b)} \sqrt{R^{2}+1-2 R \cos \theta}}{\left(R^{2}+1+2 R \cos \theta\right)^{\operatorname{Re}(-b)+1 / 2}} d \theta
\end{aligned}
$$

where $A, B, C, D$ are positive constants depending on $a, b, c$ but not on $R$.

Proof. We divide the integral into two parts according to

$$
\int_{\gamma}|F(a, b ; c ; \zeta)| \frac{|d \zeta|}{|\zeta|}=\int_{\gamma_{1}}|F(a, b ; c ; \zeta)| \frac{|d \zeta|}{|\zeta|}+\int_{\gamma_{2}}|F(a, b ; c ; \zeta)| \frac{|d \zeta|}{|\zeta|}=: \mathcal{I}_{1}+\mathcal{I}_{2},
$$

where

$$
\gamma_{1}:=\left\{\Phi\left(R e^{-i \theta}\right):|\theta| \leq \theta_{1}\right\}
$$

(the part lying in $|z-1| \leq 1 / 2)$ and

$$
\gamma_{2}:=\left\{\Phi\left(\operatorname{Re}^{-i \theta}\right): \theta_{1} \leq|\theta| \leq \pi\right\}
$$

(the part lying in $|z-1| \geq 1 / 2$ ).

In order to estimate $\mathcal{I}_{1}$ we replace $F(a, b ; c ; \zeta)$ by

$$
\begin{aligned}
F(a, b ; c ; \zeta)= & \frac{\Gamma(c) \Gamma(c-a-b)}{\Gamma(c-a) \Gamma(c-b)} F(a, b ; a+b-c+1 ; 1-\zeta) \\
& +\frac{\Gamma(c) \Gamma(a+b-c)}{\Gamma(a) \Gamma(b)}(1-\zeta)^{c-a-b} F(c-a, c-b ; c-a-b+1 ; 1-\zeta),
\end{aligned}
$$

in the case that $c-a-b$ is not an integer (see, for example, [11, pg. 113]). We thus see that

$$
\begin{aligned}
\mathcal{I}_{1} & \leq\left|\frac{\Gamma(c) \Gamma(c-a-b)}{\Gamma(c-a) \Gamma(c-b)}\right| \int_{\gamma_{1}}|F(a, b ; a+b-c+1 ; 1-\zeta)| \frac{|d \zeta|}{|\zeta|} \\
& +\left|\frac{\Gamma(c) \Gamma(a+b-c)}{\Gamma(a) \Gamma(b)}\right| \int_{\gamma_{1}}|F(c-a, c-b ; c-a-b+1 ; 1-\zeta)||1-\zeta|^{c-a-b} \frac{|d \zeta|}{|\zeta|} .
\end{aligned}
$$

Since $\zeta=\zeta(\theta)=4 R e^{-i \theta} /\left(R e^{-i \theta}+1\right)^{2}$ we have

$$
\frac{d \zeta}{\zeta}=\frac{\zeta^{\prime}(\theta)}{\zeta(\theta)} d \theta=-\left[\frac{1-R e^{-i \theta}}{1+R e^{-i \theta}}\right] i d \theta
$$

and therefore,

$$
\frac{|d \zeta|}{|\zeta|}=\left|\frac{\zeta^{\prime}(\theta)}{\zeta(\theta)}\right| d \theta=\left[\frac{1+R^{2}-2 R \cos \theta}{1+R^{2}+2 R \cos \theta}\right]^{1 / 2} d \theta
$$


From $|1-\zeta| \leq 1 / 2$ for $\zeta \in \gamma_{1}$, we obtain

$$
\begin{aligned}
\mathcal{I}_{1} & \leq A \cdot \int_{0}^{\theta_{1}}\left[\frac{R^{2}+1-2 R \cos \theta}{R^{2}+1+2 R \cos \theta}\right]^{1 / 2} d \theta \\
& +B \cdot \int_{0}^{\theta_{1}}\left[\frac{R^{2}+1-2 R \cos \theta}{R^{2}+1+2 R \cos \theta}\right]^{\operatorname{Re}(c-a-b+1 / 2)} d \theta
\end{aligned}
$$

with

$$
A:=2\left|\frac{\Gamma(c) \Gamma(c-a-b)}{\Gamma(c-a) \Gamma(c-b)}\right| \sum_{\nu=0}^{\infty}\left|\frac{(a)_{\nu}(b)_{\nu}}{(a+b-c+1)_{\nu}(1)_{\nu}}\right|\left(\frac{1}{2}\right)^{\nu}
$$

and

$$
B:=2\left|\frac{\Gamma(c) \Gamma(a+b-c)}{\Gamma(a) \Gamma(b)}\right| \sum_{\nu=0}^{\infty}\left|\frac{(c-a)_{\nu}(c-b)_{\nu}}{(c-a-b+1)_{\nu}(1)_{\nu}}\right|\left(\frac{1}{2}\right)^{\nu} .
$$

When $c-a-b$ is an integer, the estimates remain the same except for the constants involved. This can be obtained by a similar computation using the representation given in [1, pg. 559].

Now we turn to the estimate of $\mathcal{I}_{2}$. According to the reflection formula given, for example, in [11, pg. 113], we replace $F(a, b ; c ; \zeta)$ by

$$
\begin{aligned}
F(a, b ; c ; \zeta) & =\frac{\Gamma(c) \Gamma(b-a)}{\Gamma(c-a) \Gamma(b)}(-\zeta)^{-a} F(a, 1-c+a ; 1-b+a ; 1 / \zeta) \\
& +\frac{\Gamma(c) \Gamma(a-b)}{\Gamma(a) \Gamma(c-b)}(-\zeta)^{-b} F(b, 1-c+b ; 1-a+b ; 1 / \zeta)
\end{aligned}
$$

if $b-a$ is not an integer. Proceeding exactly as in the previous case and using the fact that $|\zeta| \geq \sqrt{5} / 2$ for $\zeta \in \gamma_{2}$ and $R$ so small that $\operatorname{Re} \Phi\left(R e^{-i \theta_{1}}\right)>1$, we get (for these $R$ )

$$
\begin{aligned}
\mathcal{I}_{2} \leq C \cdot \int_{\theta_{1}}^{\pi} \frac{(4 R)^{-\operatorname{Re}(a)} \sqrt{R^{2}+1-2 R \cos \theta}}{\left(R^{2}+1+2 R \cos \theta\right)^{\operatorname{Re}(-a)+1 / 2}} d \theta \\
+D \cdot \int_{\theta_{1}}^{\pi} \frac{(4 R)^{-\operatorname{Re}(b)} \sqrt{R^{2}+1-2 R \cos \theta}}{\left(R^{2}+1+2 R \cos \theta\right)^{\operatorname{Re}(-b)+1 / 2}} d \theta
\end{aligned}
$$

with

$$
C:=2\left|\frac{\Gamma(c) \Gamma(b-a)}{\Gamma(c-a) \Gamma(b)}\right| \sum_{\nu=0}^{\infty}\left|\frac{(a)_{\nu}(1-c+a)_{\nu}}{(a-b+1)_{\nu}(1)_{\nu}}\right|\left(\frac{2}{\sqrt{5}}\right)^{\nu}
$$

and

$$
D:=2\left|\frac{\Gamma(c) \Gamma(a-b)}{\Gamma(a) \Gamma(c-b)}\right| \sum_{\nu=0}^{\infty}\left|\frac{(b)_{\nu}(1-c+b)_{\nu}}{(b-a+1)_{\nu}(1)_{\nu}}\right|\left(\frac{2}{\sqrt{5}}\right)^{\nu} .
$$

When $b-a$ is an integer, then, as mentioned in the previous case, we can use the reflection formula given in [1, pg. 559] and prove that the estimates remain unchanged except for a constant. 
Remark 1. If we write

$$
\begin{aligned}
& I_{1}(R)=\int_{0}^{\theta_{1}}\left[\frac{R^{2}+1-2 R \cos \theta}{R^{2}+1+2 R \cos \theta}\right]^{1 / 2} d \theta, \\
& I_{2}(R)=\int_{0}^{\theta_{1}}\left[\frac{R^{2}+1-2 R \cos \theta}{R^{2}+1+2 R \cos \theta}\right]^{\operatorname{Re}(c-a-b+1 / 2)} d \theta, \\
& I_{3}(R)=\int_{\theta_{1}}^{\pi} \frac{(4 R)^{-\operatorname{Re}(a)} \sqrt{R^{2}+1-2 R \cos \theta}}{\left(R^{2}+1+2 R \cos \theta\right)^{\operatorname{Re}(-a)+1 / 2}} d \theta, \\
& I_{4}(R)=\int_{\theta_{1}}^{\pi} \frac{(4 R)^{-\operatorname{Re}(b)} \sqrt{R^{2}+1-2 R \cos \theta}}{\left(R^{2}+1+2 R \cos \theta\right)^{\operatorname{Re}(-b)+1 / 2}} d \theta,
\end{aligned}
$$

then we have, observing that $\theta_{1}(R) \rightarrow \arccos (1 / 3)$ as $R \rightarrow 1$,

$$
I_{1}(R) \rightarrow \int_{1 / 3}^{1} \frac{d y}{1+y} \quad(R \rightarrow 1)
$$

and in the case $\operatorname{Re}(c+1)>\operatorname{Re}(a+b)$,

$$
I_{2}(R) \rightarrow \int_{1 / 3}^{1} \frac{(1-y)^{\operatorname{Re}(c-a-b)}}{(1+y)^{\operatorname{Re}(c-a-b+1)}} d y \quad(R \rightarrow 1) .
$$

If $\operatorname{Re}(c+1) \leq \operatorname{Re}(a+b)$, then $I_{2}(R)$ tends to infinity.

Moreover, for $\operatorname{Re}(a)>0$,

$$
I_{3}(R) \rightarrow 2^{-\operatorname{Re}(a)} \int_{-1}^{1 / 3}(1+y)^{\operatorname{Re}(a-1)} d y
$$

and for $\operatorname{Re}(b)>0$,

$$
I_{4}(R) \rightarrow 2^{-\operatorname{Re}(b)} \int_{-1}^{1 / 3}(1+y)^{\operatorname{Re}(b-1)} d y .
$$

If $\operatorname{Re}(a) \leq 0$, then $I_{3}(R)$ tends to infinity and similarly for $I_{4}(R)$ in the case of $\operatorname{Re}(b) \leq 0$.

Using the abbreviation

$$
\tau(w):= \begin{cases}\operatorname{Re}(-w+1 / 2) & \text { if } \operatorname{Re}(w) \leq 0 \\ 0 & \text { if } \operatorname{Re}(w)>0\end{cases}
$$

we prove the following.

Theorem 2.3. Let $a, b, c \in \mathbb{C}$ with $c$ not being a negative integer or zero be given. Then a constant $\mu=\mu_{a, b, c}$ exists such that for all polynomials $P$ of degree $\leq n$,

$$
\|F(a, b ; c ; \cdot) * P\|_{[0,1]} \leq \mu n^{\alpha}\|P\|_{[0,1]}
$$

where

$$
\alpha:=\alpha_{a, b, c}:=\max \{\tau(a), \tau(b), 2 \tau(c+1-a-b)\} .
$$

Proof. From Lemma 1.1 we have, for arbitrary $R>1$ and $x \in K:=[0,1]$,

$$
\begin{aligned}
|F(a, b ; c ; x) * P(x)| & =\left|\frac{1}{2 \pi i} \int_{\gamma_{R}} F(a, b ; c ; \zeta) P\left(\frac{x}{\zeta}\right) \frac{d \zeta}{\zeta}\right| \\
& \leq \frac{1}{2 \pi} \int_{\gamma_{R}}|F(a, b ; c ; \zeta)| \frac{|d \zeta|}{|\zeta|} \cdot\|P\|_{[0,1] \cdot \gamma_{R}^{-1}}
\end{aligned}
$$


Moreover, since $P$ is of degree $\leq n$, by Bernstein's lemma [12, pg. 77], we get

$$
\|P\|_{[0,1] \cdot \gamma_{R}^{-1}} \leq R^{n}\|P\|_{[0,1]}
$$

(note that $1 / \Phi(z)=(1+z)^{2} /(4 z)$ maps the outer of the closed unit disk conformally onto the outer of the interval $[0,1]$ with $1 / \Phi(\infty)=\infty$ and $\gamma_{R}^{-1}=(1 / \Phi)(\{|z|=R\})$; also note that $[0,1) \cdot \gamma_{R}^{-1}$ is the interior of $\left.\gamma_{R}^{-1}\right)$.

From Lemma 2.1 we get, for arbitrary $R>1$,

$\|F(a, b ; c ; \cdot) * P\|_{[0,1]} \leq R^{n}\|P\|_{[0,1]}\left[A \cdot I_{1}(R)+B \cdot I_{2}(R)+C \cdot I_{3}(R)+D \cdot I_{4}(R)\right] /(2 \pi)$.

Now we take $R=R_{n}=1+1 / n$. Then $R_{n}^{n}<e$ and, as we have seen in Remark 1. $I_{1}\left(R_{n}\right)$ is bounded, $I_{2}\left(R_{n}\right)$ is bounded if $\operatorname{Re}(c+1)>\operatorname{Re}(a+b)$, and $I_{3}\left(R_{n}\right)$ and $I_{4}\left(R_{n}\right)$ are bounded if $\operatorname{Re}(a)>0$ and $\operatorname{Re}(b)>0$, respectively.

If $\operatorname{Re}(c+1-a-b) \leq 0$ we have

$$
I_{2}\left(R_{n}\right) \leq \int_{0}^{\pi}\left[\frac{2+1 / n}{1 / n}\right]^{2 \operatorname{Re}(a+b-c-1 / 2)} d \theta \leq M_{1} n^{2 \operatorname{Re}(a+b-c-1 / 2)},
$$

with some constant $M_{1}$ depending on $a, b, c$ but not on $n$. Here we use the fact that $R^{2}+1-2 R \cos \theta \geq(R-1)^{2}$ and $R^{2}+1+2 R \cos \theta \leq(R+1)^{2}$ when $\operatorname{Re}(c-a-b+1 / 2) \leq$ 0 .

Moreover, if $\operatorname{Re}(a) \leq 0$, we have, with a constant $M_{2}$ not depending on $n$,

$$
I_{3}\left(R_{n}\right) \leq \int_{0}^{\pi}(4(1+1 / n))^{-\operatorname{Re}(a)} \frac{\sqrt{2+1 / n}}{(1 / n)^{\operatorname{Re}(-a+1 / 2)}} d \theta \leq M_{2} n^{\operatorname{Re}(-a+1 / 2)} .
$$

This follows as a consequence of $R^{2}+1-2 R \cos \theta \leq(R+1)^{2}$ and $R^{2}+1+2 R \cos \theta \geq$ $(R-1)^{2}$. Of course, a similar argument holds for $I_{4}\left(R_{n}\right)$ if $\operatorname{Re}(b) \leq 0$. A combination of the above estimates gives the assertion for all parameter cases.

Remark 2. If

$$
\operatorname{Re}(a)>0, \operatorname{Re}(b)>0 \text { and } \operatorname{Re}(a+b)<\operatorname{Re}(c+1),
$$

then $\alpha=0$ so that there exists a constant $\mu=\mu_{a, b, c}$ with

$$
\|F(a, b ; c ; \cdot) * P\|_{[0,1]} \leq \mu\|P\|_{[0,1]}
$$

for all polynomials $P$. This result can also be obtained from the integral representation

$$
F(a, b ; c ; z) * g(z)=\int_{1}^{\infty} \lambda\left(\frac{1}{s}\right) g\left(\frac{z}{s}\right) \frac{d s}{s}=\int_{0}^{1} \lambda(t) g(z t) \frac{d t}{t}
$$

where

$$
\lambda(t)=\frac{\Gamma(c)}{\Gamma(a) \Gamma(b) \Gamma(c-a-b+1)} t^{b}(1-t)^{c-a-b} F(c-a, 1-a ; c-a-b+1 ; 1-t)
$$

(see 2]). Actually, this integral representation is valid for all functions $g$ that are holomorphic in a region $S$, being starlike with respect to 0 (and for all $z \in S$ ). Moreover, it may be viewed as the limiting case $R \rightarrow 1$ of the integrals (1.2) with $\gamma=\gamma_{R}$ as above (cf. the proof of Theorem 2 in 8 ] for the case $b=1$ ). Using (2.5), we see that the constant $\mu$ (under the restrictions (2.4)) can be taken as

$$
\mu=\int_{0}^{1}|\lambda(t)| \frac{d t}{t} .
$$


If, in addition, $a, b, c$ are real, then $\mu=\int_{0}^{1} \lambda(t) d t / t=1$ (cf. also Theorem 1 in [8] for the case $b=1$ ).

\section{Application to $M(a, c ; \cdot)$}

In this section we introduce certain polynomial expansions which can be used for the efficient evaluation of the confluent hypergeometric functions $M(a, c ; \cdot)$ for varying parameters $a, c$ on a line segment

$$
K_{\beta}:=\{t \beta: t \in[0,1]\},
$$

where $\beta \in \mathbb{C}, \beta \neq 0$. In particular, we apply Theorem 2.3 to estimate the order of magnitude of the terms and the speed of convergence of the corresponding partial sums.

The starting point is to write $M(a, c ; \cdot)$ as the Hadamard product

$$
M(a, c ; z)=F(a, b ; c ; z) * M(1, b ; z) .
$$

In the special case $b=1$, the second factor reduces to the exponential function. This situation is studied in detail in 9, 10. In our work we consider more general $b \in \mathbb{C}, b \neq 0,-1,-2, \ldots$. In fact, it turns out that this higher flexibility leads to better results for various parameters $a$ and $c$.

From [6. pg. 35] we obtain a series representation for $M(1, b ; \cdot)$ in terms of shifted Chebyshev polynomials $T_{n}^{*}$ (defined by $T_{n}^{*}(x)=T_{n}(2 x-1)$, with $T_{n}$ being the $n$-th Chebyshev polynomial) as

$$
M(1, b ; z)=\sum_{n=0}^{\infty} c_{n}(\beta) T_{n}^{*}(z / \beta) \quad(z \in \mathbb{C}),
$$

where

$$
c_{n}(\beta)=\frac{\varepsilon_{n} \beta^{n}}{2^{2 n}(b)_{n}}{ }_{2} F_{2}(1+n, 1 / 2+n ; b+n, 2 n+1 ; \beta) .
$$

Here $\varepsilon_{n}$ is 1 when $n=0$, and 2 when $n>0$. Since the $M(a, c ; \cdot)$ are entire functions, the convergence is locally uniform in $\mathbb{C}$ (with "overgeometric" rate of convergence).

Moreover, we know that the shifted Chebyshev polynomials have a hypergeometric representation

$$
T_{n}^{*}(x)=(-1)^{n} F_{1}(-n, n ; 1 / 2 ; x) .
$$

By the continuity of the Hadamard product we have

$$
\begin{aligned}
M(a, c ; z) & =\sum_{n=0}^{\infty}(-1)^{n} c_{n}(\beta)(F(a, b ; c ; z) * F(-n, n ; 1 / 2 ; z / \beta)) \\
& =\sum_{n=0}^{\infty}(-1)^{n} c_{n}(\beta)_{4} F_{3}(-n, n, a, b ; c, 1 / 2,1 ; z / \beta) \quad(z \in \mathbb{C}) .
\end{aligned}
$$

Observe that ${ }_{4} F_{3}(-n, n, a, b ; c, 1 / 2,1 ; \cdot / \beta)$ are polynomials of degree $n$. The computation of the polynomials numerically is made easy by recurrence formulas ( 6 , pg. 145]; see next section). Moreover, the coefficients $c_{n}(\beta)$ are independent of the parameters $a$ and $c$. This means that, as soon as we have fixed $\beta$ and $b$, the $c_{1}(\beta), \ldots, c_{N}(\beta)$ (for some positive integer $N$ ) may be precomputed and stored. So if we are interested in a routine for computing $M(a, c ; \cdot)$ for varying parameters, this turns out to be a major advantage compared, for example, to the (shifted) 
Chebyshev expansions of $M(a, c ; \cdot)$ on $K_{\beta}$ (where the coefficients depend on the parameters).

Since $\left\|T_{n}^{*}\right\|_{[0,1]}=1$ for all $n$, we obtain from Theorem 2.3 ,

(3.1) $\left\|_{4} F_{3}(-n, n, a, b ; c, 1 / 2,1 ; \cdot / \beta)\right\|_{K_{\beta}}=\left\|_{4} F_{3}(-n, n, a, b ; c, 1 / 2,1 ; \cdot)\right\|_{[0,1]} \leq \mu n^{\alpha}$,

where $\alpha$ is as in Theorem 2.3

This means that the sequence of polynomials $\left\{{ }_{4} F_{3}(-n, n, a, b ; c, 1 / 2,1 ; \cdot / \beta)\right\}_{n}$ is bounded on $K_{\beta}$ if $\alpha=0$ (similar to the sequence of Chebyshev polynomials on $[-1,1])$ and grows with only arithmetic speed in the case $\alpha>0$.

Let $\mathcal{S}_{n}(a, b, c, \beta, \cdot)$ be the $n$-th partial sum of the above series. Then

$$
\left\|M(a, c ; \cdot)-\mathcal{S}_{n-1}(a, b, c, \beta, \cdot)\right\|_{K_{\beta}} \leq \sum_{k=n}^{\infty}\left|c_{k}(\beta)\right| \cdot\left\|_{4} F_{3}(-k, k, a, b ; c, 1 / 2,1 ; \cdot / \beta)\right\|_{K_{\beta}} .
$$

Since

$$
\lambda:=\sup _{n, k}\left|(1+n)_{k} /(b+n)_{k}\right|<\infty
$$

(this can be shown easily by applying Stirling's formula), we have

$$
\left|{ }_{2} F_{2}(1+n, 1 / 2+n ; b+n, 2 n+1 ; \beta)\right| \leq \lambda e^{|\beta|}
$$

and therefore

$$
\left|c_{n}(\beta)\right|=\mathcal{O}\left(\frac{|\beta|^{n}}{4^{n}} \frac{1}{\left|(b)_{n}\right|}\right) \quad(n \rightarrow \infty) .
$$

Moreover, since $(b)_{n} \sim n^{b} n ! / \Gamma(b)$ for $n \rightarrow \infty$, we find

$$
\left|c_{k}(\beta)\right| \cdot\left\|_{4} F_{3}(-k, k, a, b ; c, 1 / 2,1 ; \cdot / \beta)\right\|_{K_{\beta}}=\mathcal{O}\left(\frac{k^{\alpha-\operatorname{Re}(b)}}{k !} \frac{|\beta|^{k}}{4^{k}}\right) \quad(k \rightarrow \infty) .
$$

Finally, it can be shown that

$$
\sum_{k=n}^{\infty} \frac{k^{\alpha-\operatorname{Re}(b)}}{k !} \frac{|\beta|^{k}}{4^{k}}=\mathcal{O}\left(\frac{n^{\alpha-\operatorname{Re}(b)}}{n !} \frac{|\beta|^{n}}{4^{n}}\right) \quad(n \rightarrow \infty)
$$

and thus

$$
\left\|M(a, c ; \cdot)-\mathcal{S}_{n-1}(a, b, c, \beta, \cdot)\right\|_{K_{\beta}}=\mathcal{O}\left(\frac{n^{\alpha-\operatorname{Re}(b)}}{n !} \frac{|\beta|^{n}}{4^{n}}\right) \quad(n \rightarrow \infty) .
$$

For the partial sums

$$
s_{n}=s_{n}(a, b ; \cdot)=\sum_{\nu=0}^{n} \frac{(a)_{\nu} z^{\nu}}{(c)_{\nu} \nu !}
$$

of the Taylor series of $M(a, c ; z)$ it turns out that

$$
\left\|M(a, c ; \cdot)-s_{n-1}\right\|_{K_{\beta}} \sim \frac{(a)_{n}|\beta|^{n}}{(c)_{n} n !} .
$$

So we obtain for the quotient of the absolute errors

$$
\frac{\left\|M(a, c ; \cdot)-\mathcal{S}_{n-1}(a, b, c, \beta, \cdot)\right\|_{K_{\beta}}}{\left\|M(a, c ; \cdot)-s_{n-1}\right\|_{K_{\beta}}}=\mathcal{O}\left(\frac{n^{\alpha+\operatorname{Re}(c)-\operatorname{Re}(b)-\operatorname{Re}(a)}}{4^{n}}\right) \quad(n \rightarrow \infty) .
$$

This shows a favorable rate of convergence of the partial sums $\mathcal{S}_{n}(a, b, c, \beta, \cdot)$. The main advantage of these approximants, however, consists in the essentially reduced cancellation errors compared to the Taylor sections $s_{n}$, as is seen below. 
3.1. Numerical observations. As mentioned before the main disadvantage in using the Taylor series consists in computation errors occurring in the case of fixed precision arithmetic when the function values are small in modulus compared to the modulus of the terms of the series (so-called cancellation errors). We shall see that such problems may be essentially reduced by using polynomial expansions as introduced in the above section. As an example we consider the $M(a, c ; \cdot)$ on the line segment $K_{25 i}$ on the imaginary axis (so $\beta=25 i$ now).

The polynomials ${ }_{4} F_{3}(-n, n, a, b ; c, 1 / 2,1 ; \cdot)$ are evaluated with the help of the recurrence relation given in [6, pg.145]:

$$
\begin{aligned}
n(n-3) & (2 n-5)(n+c-1) f_{n}(z) \\
=- & {\left[2(n-3)(n-1)^{2}(2 n-3)(n+c-2)\right.} \\
& -\left(2 n^{2}(n-3)(n+c-1)\right. \\
& +4 z(n+a-1)(n+b-1)(n-3))(2 n-5)] f_{n-1}(z) \\
- & {\left[(n-1)(n-2)^{2}(2 n-3)(n+c-3)(2 n-5)\right.} \\
& +n^{2}(n-3)(2 n-1)(2 n-5)(n+c-1) \\
- & 4(n-1)^{3}(2 n-3)(n+c-2)(n-3) \\
+ & 8 z(n-1)(n-3)((n+a-2)(2 n-3)(n+b-2) \\
& -(2 n-5)(n+a-1)(n+b-1))] f_{n-2}(z) \\
- & {\left[(n-4)^{2}(2 n-3)(n-c-3)\right.} \\
& -(n-3)^{2}(2 n-5)(n-c-2) \\
& +z(n-a-3)(2 n-3)(n-b-3)] 2(n-1) f_{n-3}(z) \\
- & (2 n-3)(n-c-3)(n-1)(n-4) f_{n-4}(z),
\end{aligned}
$$

where $f_{n}(z)={ }_{4} F_{3}(-n, n, a, b ; c, 1 / 2,1 ; z)$ with $f_{0}(z)=1, f_{1}(z)=1-2 a b z / c$,

$$
\begin{gathered}
f_{2}(z)=1-\frac{8 a b z}{c}+\frac{4(a)_{2}(b)_{2} z^{2}}{(c)_{2}} \\
f_{3}(z)=1-\frac{18 a b z}{c}+\frac{24(a)_{2}(b)_{2} z^{2}}{(c)_{2}}-\frac{16(a)_{3}(b)_{3} z^{3}}{3(c)_{3}} .
\end{gathered}
$$

Also a recurrence relation for the coefficients $c_{n}(\beta)$ is given by [6, pg. 35], namely

$$
\begin{aligned}
\frac{2 c_{n}(\beta)}{\varepsilon_{n}}= & \left(-1+\frac{4(n+b)}{\beta}\right) c_{n+1}(\beta) \\
& +\left(1+\frac{4(n-b+3)}{\beta}\right) c_{n+2}(\beta)+c_{n+3}(\beta) .
\end{aligned}
$$

Here the main advantage, as mentioned earlier, is that the coefficients are independent of the parameters $a$ and $c$, and therefore we can precompute them and store in an array for further calculations. We compute the coefficients using backward recursion instead of forward recursion as it is observed that the former is more stable than the latter. 
In our numerical examples we show the errors for varying parameters $a$ and $c$. In all computations we fix $z=25 i$. As an approximation of the number of significant decimal digits we define

$$
\begin{aligned}
d_{1}(a, c ; b) & =d_{1, n}(a, c ; b) \\
& =\min \left\{15,-\log _{10}\left(\frac{\left|M(a, c ; 25 i)-\mathcal{S}_{n}(a, b, c, 25 i, 25 i)\right|}{|M(a, c ; 25 i)|}\right)\right\}
\end{aligned}
$$

and

$$
\begin{aligned}
d_{2}(a, c) & =d_{2, n}(a, c) \\
& :=\min \left\{15,-\log _{10}\left(\frac{\left|M(a, c ; 25 i)-s_{n}(a, c, 25 i)\right|}{|M(a, c ; 25 i)|}\right)\right\} .
\end{aligned}
$$

The computations are carried out in double precision computation, which gives a maximal accuracy of around 16 decimal digits. Since 15 exact decimal digits are what we require we cut off the error at a level of 15 .

Figure 3.1 shows the exact decimal digits when

$$
a=0.5(0.5) 15
$$

and

$$
c=0.25(0.5) 14.75
$$

for the 200th partial sum $s_{200}$ of the power series. We have chosen the degree as 200 to ensure that the error is the result of cancellation only and so that there is no improvement achieved by increasing the number of terms $n$.

We see that the behavior of the power series is not satisfactory in most of the parameter cases considered. Therefore, it is necessary to find methods which provide a higher accuracy for the case of computation in fixed precision arithmetic, even for the price of a maybe higher computation effort compared to the evaluation of sections of the power series.

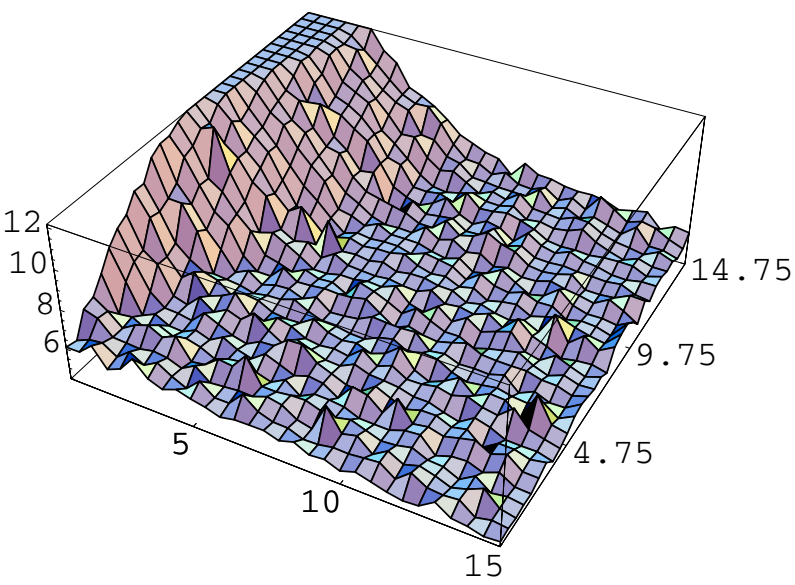

Figure 3.1. $d_{2}(a, c)$ when $a=0.5(0.5) 15$ and $c=0.25(0.5) 14.75$ 

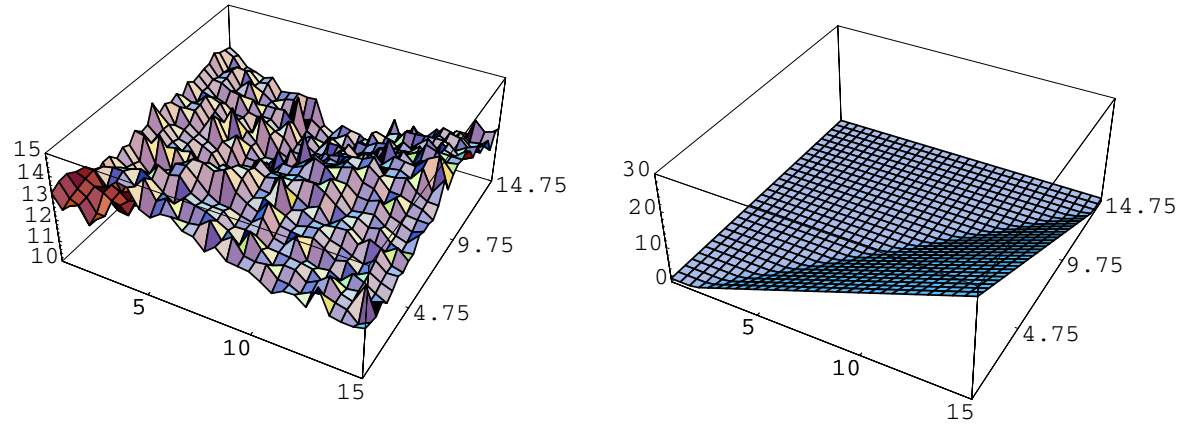

FiguRE $3.2 . d_{1}(a, c ;-0.5)$ when $a=0.5(0.5) 15$ and $c=0.25(0.5) 14.75$
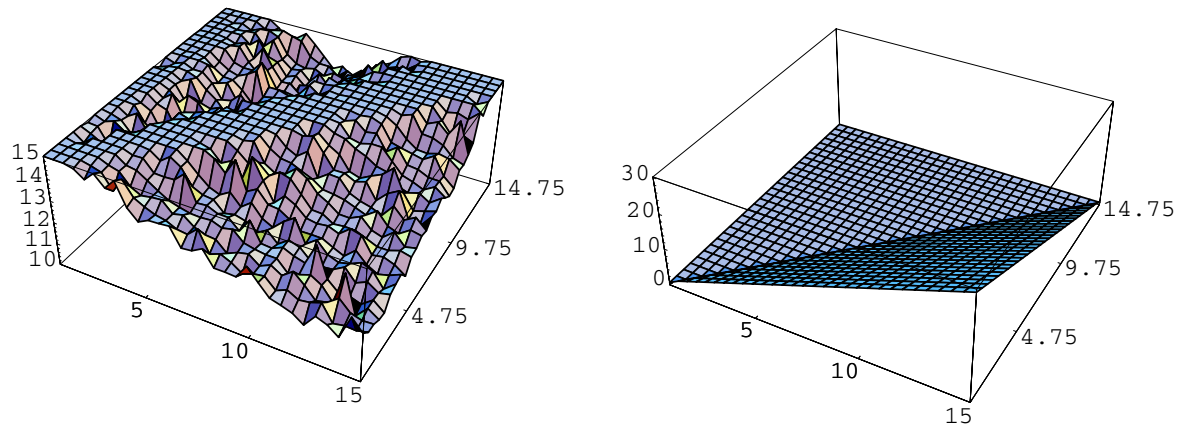

FiguRE $3.3 . d_{1}(a, c ; 0.5)$ when $a=0.5(0.5) 15$ and $c=0.25(0.5) 14.75$
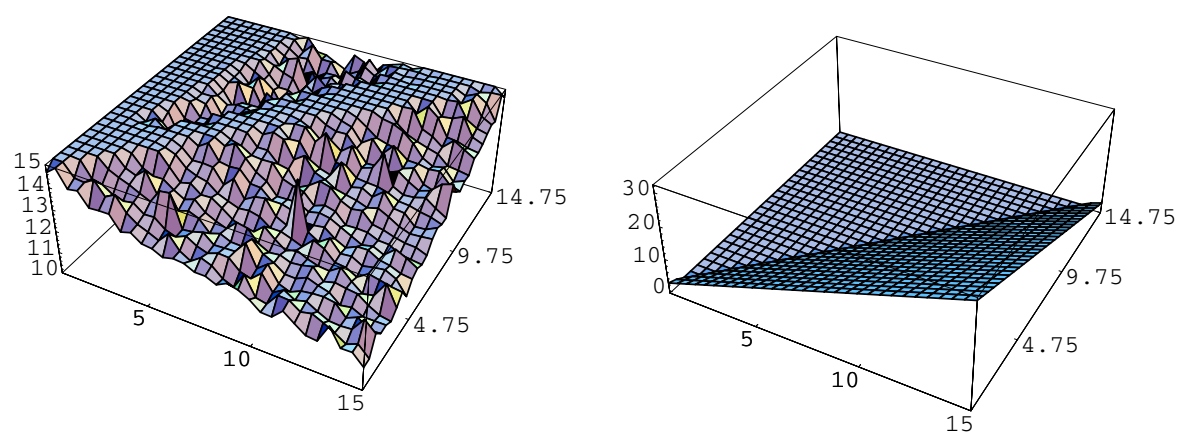

Figure 3.4. $d_{1}(a, c ; 1.5)$ when $a=0.5(0.5) 15$ and $c=0.25(0.5) 14.75$ 
Figures 3.23 .4 show the exact decimal digits for the partial sums $\mathcal{S}_{100}(a, b, c, 25 i, 25 i)$ of the series introduced above. The right-hand sides show the corresponding values $\alpha=\alpha_{a, b, c}$ from Theorem 2.3. It turns out that the cancellation problems become heavier when $\alpha$ is larger. This relates to the fact that here the polynomials ${ }_{4} F_{3}(-n, n, a, b ; c, 1 / 2,1 ; \cdot)$ and with that the terms of the series tend to be larger in modulus (cf. (3.1)). The figures demonstrate, in particular, the influence of the different choices of $b$. If $b$ is larger, then the behavior is better in the parameter regions where $c>a$. Smaller $b$ makes it possible to achieve better results in the cases $c \approx a$ and $c<a$, which turn out to be more difficult to handle.

\section{Application to $F(a, b ; c ; \cdot)$}

In this section we consider polynomial expansions for the Gaussian hypergeometric functions $F(a, b ; c ; \cdot)$ on a line segment $K_{\beta}$ with $\beta \in \mathbb{C} \backslash[1, \infty)$.

We may represent $F(a, b ; c ; \cdot)$ as a Hadamard product according to

$$
F(a, b ; c ; z)=F(a, b ; c ; z) * F(1,1 ; 1 ; z)=F(a, b ; c ; z) * \frac{1}{1-z}
$$

(note that the geometric series acts as identity with respect to Hadamard multiplication).

From [6. pg. 34] we obtain a series representation for $1 /(1-z)$ in terms of shifted Chebyshev polynomials as

$$
1 /(1-z)=\sum_{n=0}^{\infty} c_{n}(\beta) T_{n}^{*}(z / \beta) \quad\left(z \in K_{\beta}\right)
$$

where

$$
c_{n}(\beta)=\frac{\varepsilon_{n} \beta^{n}}{2^{2 n}} F(1+n, 1 / 2+n ; 2 n+1 ; \beta) .
$$

Here, again, $\varepsilon_{n}$ is 1 when $n=0$, and 2 when $n>0$.

Moreover, $T_{n}^{*}(x)=(-1)^{n}{ }_{2} F_{1}(-n, n ; 1 / 2 ; x)$ and the continuity of the Hadamard multiplication imply that

$$
\begin{aligned}
F(a, b ; c ; z) & =\sum_{n=0}^{\infty}(-1)^{n} c_{n}(\beta)(F(-n, n ; 1 / 2 ; z / \beta) * F(a, b ; c ; z)) \\
& =\sum_{n=0}^{\infty}(-1)^{n} c_{n}(\beta)_{4} F_{3}(-n, n, a, b ; c, 1 / 2,1 ; z / \beta)
\end{aligned}
$$

with the same polynomials ${ }_{4} F_{3}(-n, n, a, b ; c, 1 / 2,1 ; \cdot)$ as above.

Let $\mathcal{S}_{n}(a, b, c, \beta, \cdot)$ be the $n$-th partial sum of the above series. Then

$$
\begin{aligned}
& \left\|F(a, b ; c ; \cdot)-\mathcal{S}_{n-1}(a, b, c, \beta, \cdot)\right\|_{K_{\beta}} \\
& \quad \leq \sum_{k=n}^{\infty}\left|c_{k}(\beta)\right| \cdot\left\|{ }_{4} F_{3}(-k, k, a, b ; c, 1 / 2,1 ; \cdot / \beta)\right\|_{K_{\beta}} .
\end{aligned}
$$


Since for every $\beta \notin[1, \infty)$ we can find $\eta \in \mathbb{C} \backslash[-1,1]$ such that $\beta=2 /(1-\eta)$, we have (see [3, pg. 77])

$$
\begin{aligned}
F(n+ & \left.1, n+1 / 2 ; 2 n+1 ; \frac{2}{1-\eta}\right) \\
& =\left(\frac{4 \Gamma(2 n+1) \Gamma(1 / 2)(-\beta)^{-n-1} e^{-(n+1) \xi}}{\sqrt{n} \Gamma(n+1 / 2) \Gamma(n+1 / 2)\left(1-e^{-2 \xi}\right)}\right)\left(1+\mathcal{O}\left(n^{-1}\right)\right),
\end{aligned}
$$

where $e^{-\xi}=\eta-\sqrt{\eta^{2}-1}$ (with $\left|e^{-\xi}\right|<1$ ). Therefore, we see that, using Stirling's formula,

$$
\left|F\left(n+1, n+1 / 2 ; 2 n+1 ; \frac{2}{1-\eta}\right)\right|=\mathcal{O}\left(\frac{4^{n} e^{-n \xi}}{\sqrt{n}|\beta|^{n}}\right)
$$

and, thus,

$$
\left|c_{n}(\beta)\right|=\mathcal{O}\left(e^{-n \xi} / \sqrt{n}\right)
$$

Hence, (3.1) implies that

$$
\left|c_{k}(\beta)\right| \cdot\left\|_{4} F_{3}(-k, k, a, b ; c, 1 / 2,1 ; \cdot / \beta)\right\|_{K_{\beta}}=\mathcal{O}\left(n^{\alpha-1 / 2} e^{-n \xi}\right) .
$$

Observe that the right-hand side of the last inequality is decreasing at a geometric rate. Similarly as in the previous section this implies that

$$
\left\|F(a, b ; c ; \cdot)-\mathcal{S}_{n-1}(a, b, c, \beta, \cdot)\right\|_{K_{\beta}}=\mathcal{O}\left(n^{\alpha-1 / 2} e^{-n \xi}\right) .
$$

4.1. Numerical observations. As in the case of confluent hypergeometric functions, the main disadvantage of Taylor series of hypergeometric functions also lies in the cancellation problems occurring, in particular, when $\operatorname{Re}(a+b)$ is much larger than $\operatorname{Re}(c+1)$. Our Hadamard convolution technique helps in overcoming this problem at least for a certain range of parameter values.

The polynomials ${ }_{4} F_{3}(-n, n, a, b ; c, 1 / 2,1 ; \cdot)$ are computed as in the previous section by the recurrence formula (3.2). The coefficients $c_{n}(\beta)$ are now calculated, using a recurrence formula given in [6, pg. 34],

$$
\begin{aligned}
\frac{2 c_{n}(\beta)}{\varepsilon_{n}}= & \left(-2(n+1)+\frac{(2 n+3)(n+2)}{n+1}+\frac{4(n+b)}{\beta}\right) c_{n+1}(\beta) \\
& +\left(2 n+3-\frac{2(n+2)^{2}}{n+1}+\frac{4(n+2)}{\beta(n+1)}\right) c_{n+2}(\beta)-\left(\frac{n+2}{n+1}\right) c_{n+3}(\beta) .
\end{aligned}
$$

We again use the backward recurrence instead of the forward as it is more stable. Here the initial values are taken from the functions built into MATHEMATICA(4.1). As in Miller's algorithm [11, pg. 343], if there is a possibility of finding a normalizing series, the initial values can be replaced by any arbitrary numbers, not all of them zero. (This applies to the case of confluent hypergeometric functions also.) 

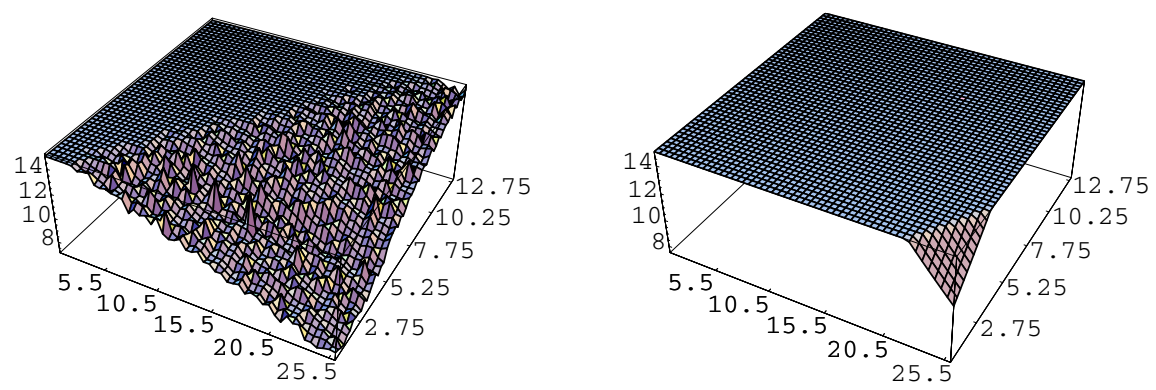

FiguRe 4.1. $d_{2}(a, c ; 2.5)$ and $d_{1}(a, c ; 2.5)$ when $a=0.5(0.5) 25.5$, $c=0.25(0.25) 12.75$

In our numerical example we take $\beta=i / 2$. Let

$$
d_{1}(a, c ; b)=\min \left\{15,-\log _{10}\left(\frac{\left|F(a, b ; c ; i / 2)-\mathcal{S}_{n}(a, b, c, i / 2, i / 2)\right|}{|F(a, b ; c ; i / 2)|}\right)\right\}
$$

and

$$
d_{2}(a, c ; b)=\min \left\{15,-\log _{10}\left(\frac{\left|F(a, b ; c ; i / 2)-s_{n}(a, b, c, i / 2)\right|}{|F(a, b ; c ; i / 2)|}\right)\right\},
$$

where $s_{n}(a, b, c, \cdot)$ is the $n$-th partial sum of the Taylor series of $F(a, b, c ; \cdot)$. Here also, for the reason as in the previous section, we cut off the error at the level 15 . Figure 4.1 shows the errors when $a=0.5(0.5) 25.5$ and $c=0.25(0.25) 12.75$ and $b=2.5$ is fixed. Here $z=i / 2$ is also fixed.

We have taken the degrees for $\mathcal{S}_{n}$ and $s_{n}$ as 60 and 200, respectively, to ensure that the error is purely because of cancellation and not of truncation of the terms of the series.

We can observe that the behavior of the power series is not satisfactory when $c+1<a+b$. In the case of the new series we obtain much better results at least for a certain strip with $c+1<a+b$.

\section{REFERENCES}

1. M. Abramowitz and I. Stegun, Handbook of mathematical functions, Dover Publ., New York, 1968. MR0208798 (34:8607)

2. R. Balasubramanian, S. Ponnusamy, and M. Vuorinen, On hypergeometric functions and function spaces, J. Comp. Appl. Math., 139(2002), 299-322. MR1876641(2003a:33011)

3. A. ERdÉLYI, Higher transcendental functions, Vol. I of Bateman manuscript project, McGraw Hill, New York, 1953. MR0698779 (84h:33001a)

4. K.G. Grosse-Erdmann, On the Borel-Okada theorem and the Hadamard multiplication theorem, Complex Variables, 22(1993), 101-112. MR1277015(95j:30002)

5. Y.L. Luke, The special functions and their approximations, Vol.I, Academic Press, 1969. MR0241700 (39:3039)

6. Y.L. LuKE, The special functions and their approximations, Vol.II, Academic Press, 1969. MR0249668(40:2909)

7. J. MüLLER, The Hadamard multiplication theorem and applications in summability theory, Complex Variables, 18(1992), 155-166. MR1157924 (93g:30004) 
8. J. MülLER, Convergence acceleration of Taylor sections by convolution, Constructive approximation, 15(1999), 523-536. MR.1702803 (2000i:41040)

9. Сн. Schwarz, Computation of confluent hypergeometric functions and application to parabolic boundary control problems, Ph.D Thesis, University of Trier, 2001.

10. CH. Schwarz, Computation of confluent hypergeometric functions on compact intervals, In: Functions, Series, Operators (Budapest, 1999), 357-366, Janos Bolyai Math. Soc., Budapest, 2002. MR.1981575 (2004g:33012)

11. N.M. Temme, Special Functions: An introduction to the classical functions of mathematical physics, John Wiley, 1996. MR.1376370 (97e:33002)

12. J.L. WALSH, Interpolation and approximation by rational functions in the complex domain, 5th ed., Amer. Math. Soc. Colloq. Publ., Providence, RI, 1969. MR0218588 (36:1672b)

University of Trier, FB IV, Mathematics, D-54286 Trier, Germany

E-mail address: luh@uni-trier.de

University of Trier, FB IV, Mathematics, D-54286 Trier, Germany

E-mail address: jmueller@uni-trier.de

Department of Mathematics, Indian Institute of Technology, IIT-Madras, Chennai600 036, INDIA

E-mail address: samy@ittm.ac.in

Department of Mathematics, Indian Institute of Technology, IIT-Madras, Chennai600 036, INDIA

E-mail address: vasu2kk@yahoo.com 